\title{
Bring Your Own Mobile Device (BYOD) to the Hospital: Layered Boundary Barriers and Divergent Boundary Management Strategies
}

\author{
Keri K. Stephens, PhD \\ The University of Texas at Austin \\ keristephens@austin.utexas.edu \\ Meena Iyer, MD \\ Seton Healthcare Family \\ miyer@seton.org
}

\author{
Yaguang Zhu, MA \\ The University of Texas at Austin \\ yaguang.zhu@utexas.edu \\ Terrie Hairston, MS, RN-BC, FACHE \\ Seton Healthcare Family \\ thairston@seton.org
}

\author{
Millie Harrison, MA \\ The University of Texas at Austin \\ millieharrison@utexas.edu \\ John Luk, MD \\ UT Austin Dell Medical School \\ john.luk@austin.utexas.edu
}

\begin{abstract}
This study examined how one US hospital implemented a mobile communication app to improve workplace communication. The hospital did not provide the technology, instead they asked their workers to use their own personal mobiles at work, through a permissive bring your own device to work (BYOD) policy. Using boundary theory, we conducted a constant-comparative analysis to examine the layers of boundary management issues. At the organizational level, the key issues were policy legacy, communicating the policy, control, dead zones, and mobile costs. At the group level, different hospital units created their own formal and informal policies. At the individual level, themes included personal mobile device use, job role expectations, and decision-making autonomy. The discussion presents examples of how healthcare workers enacted segregator and integrator boundaries. Our findings explain why it is not easy to tell hospital employees, "Go ahead and use your mobiles for patient care," and have them embrace this practice.
\end{abstract}

\section{Introduction}

Mobile devices have become the norm for communicating in our personal lives, and they are increasingly becoming the primary way to communicate at work. These devices play such an integral role in peoples' lives that they are with us at all times $[1,2]$. Yet as these devices enter our workplaces, two key boundary concerns have emerged: the blurring of work/life boundaries [3-5], and the blurring of enterprise control and personal control over mobile use $[6,7]$. While there is now over a decade of research on mobiles blurring work and non-work roles, we know considerably less about how workers respond when their companies and organizations institute policies concerning how, when, and where individuals can use their mobile devices at work.

Bring Your Own Device (BYOD) to work is a concept used in the US to describe how employees have brought their personal mobile devices to work, and information technology (IT) departments have responded. This phenomenon is often called enterprise IT consumerization because the consumers of technology-mobiles in this case-have brought their personal devices into the enterprise [6,7]. IT departments realize they must balance a company's need to control risks while capitalizing on the benefits of IT consumerization; therefore, policies and norms for appropriate mobile device use have emerged. One type of BYOD policy is a restrictive mobile device policy that bans personal mobiles $[8,9]$. These scholars studied janitorial and fast food workers who could not use their mobiles at work except in the case of emergencies. They found several unintended consequences resulting from this organizational policy, including having no way to micro-coordinate work and being inaccessible in emergencies.

The current study advances the research on organizational mobile use by examining another side of BYOD policies; what happens when people are encouraged to use their mobile devices at work? To answer this question, we rely on boundary theory [10$14]$ and focus on a context where workers are highly mobile within a single facility: a hospital. A hospital provides an ideal context to study permissive BYOD policies because there are a variety of workers who need to micro-coordinate their activities, and past research shows that mobiles facilitate microcoordination well [15]. Furthermore, hospitals are actively improving collaborative team-based care, and communication is key for team success [16]. Mobile phone use in hospitals can increase access to medical references, clinical tools, and patient information [17]. Although many hospitals now allow the integration of mobile devices into work routines to assist in 
coordination processes, it is unclear which types of hospital professionals can or should use mobile devices at work. For example, in a recent survey of over 450 healthcare organizations across North America, 73\% of the organizations supported some form of BYOD use at work [18]. While 91\% of those policies allowed physicians mobile device access, only 51\% of the policies allowed nurses that same access.

\section{Boundary Theory}

The theoretical perspective we used to guide this research is boundary theory. Boundary theory is predominantly used in management research, and it explains how people enact different role identities as they transition between social domains [10-14]. Much of this research focuses on changes between work and home boundaries, but there are also role transitions at work for issues like being a supervisor at one time and a colleague at another [10]. A key finding in the boundary literature is that people fall on a continuum from segregator to integrator concerning how they enact their roles. Some people segment their different roles by making clear demarcations between activities within each role, such as a manager not talking about her children at company events.

Boundary theory is especially helpful in contexts where people use technology to communicate across their roles. For example, now that BYOD expectations and policies exist in many US workplaces [6,19], employees often use their personal mobile devices for both business and non-business purposes. This practice can create a situation where organizations have to ask permission to access personally-owned devices [20], and there is clear boundary-blurring potential $[3,5,7,21]$. This integration of life roles through a single mobile device can diminish spatial, temporal, and social-role boundaries [13].

\section{Mobility Work}

This study applies boundary theory beyond the study of traditional knowledge workers and into the realm of mobility work in a hospital. While work is often considered co-located or distant, there is a middle ground that includes workers who are locally mobile because these workers are constantly on the move [22]. This intermediate place of work defines hospitals quite well because regardless of the job, the vast majority of hospital workers interact with patients or other caregivers daily.

There are four aspects of mobility work: resources, places, knowledge, and persons [22]. Of particular interest to the current study is the person aspect, which can be sub-divided into role and specific person. Peoples' job roles can require them to be mobile as individuals or as a group. People who are locally mobile have to balance unique sets of contradictory concerns, some of which involve technology. For example, physicians want to be available yet secluded, because they face constant interruptions if they are available all the time. Mobility and flexibility is the next balance because when people and objects are mobile, they are also difficult to locate [22]. The final balance to achieve is between orderliness and flexibility. In mobile work, orderliness is vital, but if the structures are too rigid, they are not flexible enough to be useful. In mobility work, like a hospital, action is both temporal and spatial. People change locations constantly.

In their work on mobility and mobiles, Pink and colleagues studied healthcare workers who visited patients in their homes [23]. These workers' organization provided them a mobile phone for work, and those workers also had a personal mobile phone. However, they also found that more senior field workers were given phones with Internet access and the latest scheduling tools, while more junior workers' were provided phones with very basic functions. Ironically, the more junior workers often had advanced features on their personal mobile devices despite being provided a very simple work phone. This created an awkward blurring of boundaries between personal and professional life and led to challenging decisions concerning using work-provided phones of personal devices.

\section{Interprofessional Roles and Mobiles}

Recently, researchers in the medical field have begun investigating how mobile devices facilitate interprofessional communication. This form of coordination of patient care between people who have been trained in different professions is often called interprofessional communication. Hospitals have a long history of using pagers to address local mobility issues because pagers are relatively inexpensive and function as a call alert system [24]. Yet several recent studies suggest that mobiles do more than simply replace pagers for healthcare professionals [25-27]. Specifically, mobiles decrease wait times for responses, thus improving the pace of medical decision-making [16,27]. Mobiles also empower healthcare professionals by creating a digital trail, increasing perceptions of request legitimacy [28] and helping prioritize tasks [27].

In addition to their benefits, research also suggests that using mobiles in hospital settings creates new 
problems for healthcare professionals. Physicians complain of increased interruptions [24,26,29], which can lead to heightened perceptions of overload. Many hospital professionals also claim that mobiles reconfigure their communication patterns such that there is less face-to-face conversation and they lose important contextual information [16,24,28]. Moreover, the fact that most smartphones have built-in cameras also raises concerns on patient privacy under the Health Insurance Portability and Accountability Act (HIPAA) of 1996 [30]. Finally, researchers have yet to find direct evidence for how mobile devices improve clinical management and reduce safety concerns [31]. Thus, although previous studies have identified some of the advantages and disadvantages of mobile devices in hospital settings, much remains unclear [32].

\section{Study Research Questions}

Extant research exposes a gap in our understanding of how mobiles impact hospital communication. In this current study, we examined a hospital implementing personal mobile devices at work. BYOD policies are growing in popularity $[6,7,18]$. By using boundary theory and the levels of boundaries involved in organizational mobile device use, we ask the following guiding research question:

RQ: When an organization enacts a BYOD policy in a hospital that allows personal mobile device use, what boundary management issues emerge at the individual, group/team, and organizational levels?

\section{Method}

\subsection{Study Site}

This study was conducted in 2015, and our team acquired IRB approval as an exempt study from the University and the hospital IRB. The site chosen for this study was a 248 bed hospital in a large metropolitan city in the southwestern US. We chose this location because the hospital uses many communication technologies and has a history of implementing technology like facility-wide electronic health records in 2012. Their recent implementation of a mobile application that complied with HIPAA privacy and security standards in the US is our focus here.

This hospital is part of a larger network of hospitals. In Spring 2014, the hospital system approved the use of this HIPAA-compliant text-messaging app (called HIPAA-Text in this study), and they began a pilot program that included only physicians. After a six- month trial, the hospital purchased enough licenses that all the staff could access this app. While app training occurred, the hospital system removed monetary support for the physicians' and residents' personal mobile phones. A year and half later, over $70 \%$ of the physicians used HIPAA-Text, but only $30 \%$ of their nurses used this tool.

\subsection{Data Collection Process}

This case study used several data collection methods to achieve a comprehensive understanding of mobile device use. First, we accessed archival data on HIPAA-Text use by different professionals in this hospital. Second, we did 25 hours of focused observations examining the workflow of nurses and physicians. Third, we attended a workshop on the topic of mobile communication where staff members were encouraged to interact with members of other professions. Observing this workshop led us to conduct focus groups where participants were separated by profession to allow for more open conversation about different ways that professions communicate. There were a total of four nursing focus groups and four physician/resident focus groups. In addition, members of our team participated in an ongoing interprofessional communication task force. Together, these data-gathering activities produced 250 pages of double-spaced text used in this analysis.

In the four nursing focus groups, there were a total of 22 participants representing managers, charge nurses, floor nurses, and night-shift nurses. The participants varied from experienced nurses with over 25 years of practice to nurses just entering the field with less than one year of experience. Nurses were predominantly female. In the four physician focus groups, there were a total of 24 participants with approximately equal numbers of males and females represented. These groups also varied in their race/ethnicity and their experience ranged from over 40 years to staff in their first year.

When the participants arrived for the focus group, they were given an informed consent document and asked to select a pseudonym since the session would be recorded. The focus group schedule contained 17 primary questions and 12 of them were used to answer the specific research question of this study. Our first questions asked participants to describe how they used communication media and devices at work. We followed with questions comparing how they communicate with members of their same profession and those different from them. We asked about knowledge of policies, using technology in front of patients, how privacy rules and norms impact mobile use, and barriers to mobile use. 


\subsection{Data Analysis}

The first step of analysis generated 235 open codes in the nurse focus groups, 202 in the physician focus groups, and 174 in the physician/resident focus groups that described the data. One author did the detailed coding for nurses and a separate author coded the physicians. Two of the additional authors double checked and verified the comprehensiveness of the codes. Next, the researchers began to "separate, sort, and synthesize... data through qualitative coding" [33], followed by a constant comparison [34], where each category was organized based on its theoretical and practical similarity to the other categories.

While comparing the data, we were also cognizant of our research question and how it involved three levels of analysis: individual, group/team, and organization. Since we were approaching the analysis on multiple levels, we met with a working task force to report our early findings and conduct a member check [33]. Finally, all authors reviewed the primary categories, the examples in each category, and collectively generated the theoretical interpretation of the results described next.

\section{Results}

This study addressed the following research question: When an organization enacts a BYOD policy in a hospital that allows personal mobile device use, what boundary management issues emerge at the individual, group/team, and organizational levels? Our results indicate that boundary management issues vary widely between nurses and physicians on the individual and group/team levels. Furthermore, at the organizational level, it is more difficult to reach nurses with information concerning mobile device use policies-a contributing factor to why adoption varies by organizational level as well as by organizational role. We present these results in sections focused on levels of analysis. Since the organizational issues set the context for many interpretations at lower levels, we first discuss this level of analysis. The discussion of these findings helped us create two models (see Figures 1 and 2) that contrast mobile boundary management between boundary integrators and boundary segregators. The discussion also elaborates the theoretical and empirical explanations for why mobile device policies vary in their enactment across macro, meso, and micro organizational levels.

\subsection{Organizational-Level Boundary Issues}

While this hospital decided to implement a BYOD policy and promote the use of a HIPAA-compliant mobile app, we identified five overarching barriers that inhibited adoption: policy legacy, policy-related communication, organizational control, infrastructure, and costs.

6.1.1. Policy Legacy. There was a murky understanding of mobile device policies based on a gap in health professionals' perceptions of what was an old and what was a current policy. Nurses commented, "We're not supposed to use a personal phone," and "Hospital wide, there's absolutely a policy regarding phone use." In the data, health professionals often referenced what they thought was current policy, but they were not aware that the policies had changed. Furthermore, in the past some of these health professionals signed agreements saying they would not use their personal mobiles at work. Since it would be odd to have them sign saying they can use their mobiles at work, their memory was stronger concerning policies where they signed. We refer to this lingering misconception as a legacy created by policies.

Policy legacy is not a concept obvious to people in this organization, and it only emerged as our team triangulated the official organizational documents with our focus group and observational data. Historically, hospitals had no way to communicate patient data privately through mobile devices, so it makes sense that health professionals are conservative and slow to adopt practices that have previously been taboo. In some ways, the policy legacy created a buffer between perceptions of operational correctness, personal preferences, and the newly-issued BOYD policy. People who preferred to avoid using their mobiles could simply say they thought there was policy against its use. However, policy legacy also lead to confusion on the boundaries of acceptable mobile device use.

This confusion was most obvious when health professionals discussed communication practices and the fact that they communicate two types of information: HIPAA-regulated private information and generic information containing no private patient information. For example, one nurse said, "There is an administrative policy that prohibits texting patient information back and forth to physicians. And there's nothing said about just texting, but if it contains patient information, then you are not supposed to do it. That's where HIPAA-Text comes up. It might be the official way to communicate with physicians." A charge nurse explained her understanding (a misunderstanding) of the policy this way: "Hospital wide, there's absolutely a policy regarding phone use. We are not to have our phones out, we're not to be using them in patient care 
areas. Specifically, they did a push about not walking and using your phone, because people kept hurting themselves. [laughter] There was a legitimate line there. We had to sign saying that we were not going to walk and text at the same time, because so many people hurt themselves.”

6.1.2. Communicating the policy. Despite organizational efforts to communicate the acceptability of using HIPAA-Text and personal mobile devices, many health professionals-especially nurses-were still uncertain. One nurse explained this dilemma well: "I don't use my own personal cell phone because it has my own personal email on it, has my own personal Facebook on it, and I don't know what privacy laws are and things like that, but using your own device for work kind of sets you up for-if something ever happened or went down or whatever and you're using your own personal device, that kind of opens up your own personal life to scrutiny.”

Even though health professionals were allowed to use their personal mobile devices, it was still hard for the organization to communicate the BYOD polices with them because there was a legacy of concern about privacy and personal mobile phone use in a hospital.

In addition, the diffusion and implementation process of HIPAA-Text was perceived as slow. One nurse explained, "Some units have HIPAA-Text, but not all the nurses were able to get signed up with HIPAA-Text when they were here last year, and they only came like once or twice through the whole year to sign people up.” By examining the organizational documents, our team learned that the hospital had at least five different visits from this vendor, yet most of the early visits were specifically focused on physicians. This is because the implementation was phased and during the pilot phase, the hospital only had enough licenses to allow physicians to use HIPAA-Text. Now that the hospital wanted to roll the app out to all clinical personnel, many hospital professionals still operated with the early roll-out knowledge.

6.1.3. Organizational control needs. Another misunderstanding between the organization and the health professionals concerned the requirements for using a personal mobile device, and the various applications, at work. For example, if health professionals wanted to check work email on their personal mobiles, they needed to download encryption software that allowed the organization to wipe data from a personal mobile if it was ever lost or stolen. That was not the policy if workers used HIPAA-Text because no data was stored on personal mobiles. Yet health professionals were not clear concerning the differences, and many of them used this as a reason to avoid implementing HIPAA-Text. For example, one physician shared his understandings on HIPAA-Text with others who showed apparent confusion on the encryption topic: "You can use HIPAA-Text and text and not have that encryption software. But if you get work email on your personal device, you have to have it." This was the only person in any focus group to have a clear understanding of encryption requirements.

6.1.4. Infrastructure dead zones. In this hospital, almost every health professional who participated in our study reported having issues with mobile phone reception. One physician explained, “There are certain residents whose phones just do not work in the hospital, and the only way you can get a hold of them is to have the operator page them to your number." Likewise, a resident mentioned, "The service is really poor for a lot of the different service providers, so you don't get calls. You drop calls. You don't get messages." In the same vein, one nurse said she was not able to use her personal cell phone to contact her charge nurse or physicians because most the time she did not have good reception. The term "dead zones” was repeatedly mentioned by hospital professionals as being a key issue when using mobile devices and the HIPAA-Text app.

Dead zones-real or perceived-created serious boundary management issues for interprofessional communication at this hospital. In every focus group, over $80 \%$ of the participants mentioned dead zones, so this was likely a very real issue in this organization. Since the mobile-app technology was considered erratic, health professionals used the perceived unreliability as an excuse for delayed responses to other hospital workers.

6.1.5. Employees bearing mobile costs. The final organization-level issue was also related to legacy policies and perceptions of differential treatment. Until two years ago, physicians were reimbursed for using their personal mobile devices. After the implementation of the BYOD policy, no one was reimbursed for their mobile phone use, but many people did not realize this change. The organization offered a slight discount on a data plan if employees chose to use the preferred provider, but our data suggest that this provider did not work well in many parts of the city. There was a boundary issue concerning plan choice and whether employees chose a plan that functioned well at work or one that allowed mobile access in peoples' homes. One resident explained that the best option for reliable service was to "get the provider that's in the hospital, because it eliminates so much of your hassles as an intern. But you're going from a bill that's maybe $\$ 16$ or $\$ 30$ to a 
bill that's $\$ 97$ to $\$ 100$ per line.” In this city, the average mobile plan with a data packages costs approximately $\$ 70$ a month, or $\$ 849$ a year [35].

\subsection{Group-Level and Team-Level Boundary Management Issues.}

What the organization in this study did not anticipate is that even if they issued an organizationwide BYOD policy, groups and hospital care units would create their own formal and informal rules. As workers discussed their perceptions of policies, it was obvious that some units had specific policies, and those policies were often profession-specific. For example, in one unit a formal written policy was created that banned nurses from using their personal phones at work. As one nurse shared, "We have physicians [who] will use HIPAA-Text. But as a unit, we don't use HIPAA-Text.” One respondent explained that in the past, people in her unit were seen using social media for personal use and the supervisors decided that mobile phones were a distraction and not a work tool.

Differential rules in diverse units of the hospital became obvious when we spoke with nurses and physicians who were not stationed in a single unitworkers we call ultra-mobile. These ultra-mobile workers were quite aware that the rules varied depending on where they worked. The nurses who were ultra-mobile became conservative in their mobile device use, rarely using HIPAA-Text or checking their email on their mobile devices. These ultra-mobile nurses decided it was easier to eliminate their mobile use rather than risk being seen as using them inappropriately.

The ultra-mobile nurse practitioner adopted a very different perspective. She shifted $100 \%$ of her communication to her mobile device because she believed those policies only applied to the nurses. She explained that her mobile phone helped her maximize her efficiency by being available for communication at all times and in all places.

Units also varied in how they conducted scheduling of shifts, which played a role in how people used their mobiles. Some units-especially physicians and nurses who work in administration-said that email was good way to schedule meetings. Staff nurses, on the other hand, rarely checked email. For these nurses, email was not a timely form of communication, and most email messages were irrelevant. To facilitate scheduling, staff nurses instead experimented with social media platforms like Yammer and Facebook because they wanted to reach one another with last minute schedule changes. As one nursing supervisor explained, "We created a Facebook group and there's nothing patient-specific on there, but people put schedule request changes there.” Several units also used texting. One nurse explained that texting was less invasive when it came to scheduling: "There is certain info... I would be okay being texted, 'Hey, we're having a debrief tomorrow morning, I'd love for you to come. We really need extra nurses for night shift tomorrow.' You know, stuff like that is fine.”

\subsection{Individual-Level Boundary Issues.}

Zooming into the individual-level boundary management issues, we found health professionals' personal preferences were often in conflict with organizational BYOD polices and team/unit BYOD norms. Individual preferences mirrored the boundary literature in that they were integrators who used personal mobiles for work and home roles, or they were segregators who keep their personal and professional lives distinct. Furthermore, this preference moved beyond personal/professional boundaries and into specific work roles. For example, health professionals repeatedly discussed their individual preferences on mobile device use in particular contexts, such as in the presence of patients or when making rounds.

Compared to nurses, physicians had more autonomy and flexibility in terms of mobile phone use, which led to different boundary management issues. One physician said she had two mobile phones: one for personal use (including social media, text message, and phone calls) and one chief-resident phone for work (including text messages, phone calls, and emails). Another physician pointed out he used his personal mobile for both work and personal issues. Nurses, on the other hand, did not articulate this kind of boundary control. Since individual-level boundary management issues differed by specific profession, next we discuss two structural explanations that affected how nurses viewed mobiles: nursing hierarchy and patient-facing work.

6.3.1. Nursing hierarchy. Nursing practice involves a hierarchical set of job roles with units having charge nurses, nurse practitioners, or nurse educators. Those nurses who are on the top of nursing hierarchy have more power in terms of who they directly contact through mobiles and other communication tools. For example, when asked about the accessibility to HIPAA-Text, one floor nurse said: "The doctors all have HIPAA-Text. The nurses don't. Charge nurses do, but you can't go hunt your charge nurse down for every little order that you need and have her HIPAAText the doc." This example illustrated how the nursing hierarchy erected barriers that prevented direct communication between physicians and floor nurses. 
6.3.2. Patient-facing work. Staff nursing is a job with a high degree of patient-facing work. These nurses typically spent more time communicating with patients directly face-to-face compared to other health professionals (e.g., physicians). Nurses and other professionals often commented that it was less appropriate for nurses to use their personal mobiles in front of patients than it was for physicians. One nurse supervisor explained: "I often wonder what families are thinking when they see me on my personal cellphone. Because they cannot see the screen, they do not know what I'm doing. Even if I'm just sitting at my desk on the program for HIPAA-Text, what's their perception?”

Several nurses said that they needed to use the calculator function of their personal mobiles because “...it's a very helpful tool. But then I know people think that I'm texting or on Facebook or something." To overcome this perception, nurses verbally explained why they were using their personal mobiles and sometimes showed their screen to the patient. Nurses also mentioned that mobile phones can interfere with their sense of professionalism. Beeping, ringing, and singing ring tones were a nuisance, and the tendency for nurses, hospitalists, or residents to answer their phones in the middle of a consultation was viewed as irritating and unprofessional.

\section{Discussion}

This study is one of the first to examine how organizations can influence mobile device use (see 8, for an example of how organizations keep personal mobile devices out of the workplace). A key contribution of our study is that we dove deeply into understanding how policy legacy affected adoption of mobile devices. While it is common to pilot test new apps, software, and systems in organizations before introducing the change to the entire organization, we showed how the piloting in one specific professional group-physicians in our study-created an adoption divide. In this case, the physicians chosen as the pilot test group for HIPAA-Text, represented a profession often viewed as privileged members of the hospital ecosystem [36]. It would surprise few people that physicians could use mobile tools more freely than a profession like nursing because physicians often function more autonomously than nurses [36]. In this way, our study responds to calls for research that considers the real power differentials found between hospital professions, especially when examining technology designed to facilitate communication [37].

Yet increasingly physicians and nurses need to communicate more effectively and efficiently to provide quality patient-centered care [16]. When most physicians have adopted a mobile app like HIPAAText and the nurses have not, it creates a challenging barrier for communication. Since the physicians piloted the app, there is an added burden on the organization as they try to communicate a permissive BYOD policy that includes nurses. In this organization, most nurses worked shifts, and it was difficult to get some or all of them together to share new policies. Furthermore, it was not uncommon for people in this organization to work three to four days and then be off duty for seven to eight days. Finally, keeping track of who has received notifications can be problematic. The responsibility to share policy changes often falls on the shoulders of the supervisors.

Other organizational-level issues included the reliability of the mobile tools and apps that the hospital was promoting, as well as who paid for mobile plans. Mobile connectivity is crucial for interprofessional communicators who need to coordinate workflows, exchange information in real time, and engage in collective decision-making processes. However, the dead zones frequently discussed in this data seriously impeded perceptions of reliable mobile communication. Specific sites like the lunchroom and the resident's work area got limited reception; thus, the nurses and physicians openly acknowledged that their mobile system was erratic. The permissive BYOD policy did not matter because the teams believed that using mobile was unreliable.

\subsection{Integrating Boundary Issues: Integrators or Segregators and Mobile Use}

In addition to adding an organizational perspective into this analysis of workplace mobile devices, a second contribution is our multi-level analysis. Our investigation aligned with a systems approach [38], which provides an appropriate framework to develop an integrative understanding of boundary management issues concerning BYOD-one that incorporates multiple levels of analysis. A systems approach examines the system as a whole instead of focusing only on one level of analysis. This perspective captures the complexity inherent in organizations and examines interdependent interactions among system parts [39], like different hospital professions.

In this study, we used boundary theory to identify patterns that people used to integrate or segregate their roles. To understand how mobiles functioned in these boundary patterns, we elaborate on two examples: Anika, a nurse practitioner who has fully integrated all her work roles and personal roles and uses her personal mobile as her only communication tool. We also discuss Liz, a nurse who works between units and uses 
her personal mobile to completely segregate her work and personal life. Anika is an example of what we call an organizational boundary collapser, someone who is willing and able to make organizational, group, and individual boundaries completely overlap. On the other hand, Liz's individual-level decision to solidify boundaries between her work and personal life drive her decisions to avoid using her mobile at work. We discuss each of these extended examples next.

7.1.1. Anika, the Boundary Integrator. "Nobody reimburses me for my iPhone use, but I'm all over the hospital and I use it for everything." Anika is a nurse practitioner whose job has her seeing over 30 different patients a day located in 10 to 15 different hospital units. She goes where the hospital needs her, and she is always moving. Since she is using her personal mobile phone constantly at work, she has no problem answering personal calls on that same device, and she weaves her personal conversations between her work responsibilities. She has been a nurse practitioner for over 15 years, and she explains that nothing overloads her because she has been doing her job for so long. She knows how to be efficient, and her mobile defines efficient communication for her.

Anika has a unique boundary-spanning role at this hospital and as a person who is comfortable integrating her work and personal roles, she has boundaries that are so permeable she has collapsed them. Furthermore, her mobile use is supported at all three organizational levels. She is not directly tied to any specific hospital unit, so she does not feel the need to abide by any unitspecific rules. Anika integrates her personal and professional lives, and her role supports her decision to enthusiastically embrace mobile use in this hospital. See Figure 1 for an example of how a boundary integrator uses a mobile device in hospital work.

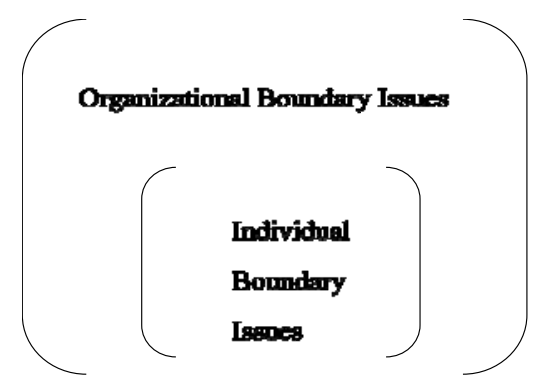

Figure 1. Boundary integration and mobile use

This figure illustrates how workers who have collapsed organizational, unit, and individual-level boundaries function in an environment where personal and professional life intermix. There are still organizational and individual-level concerns, but the boundaries are permeable.
7.1.2. Liz, the Boundary Segregator. "I go to the different areas. Where is it approved and where is it not? And what if I drag this thing out and the supervisor comes by and says, 'What are you doing on your phone?'” As a nurse, Liz is more bound by unitlevel formal and informal policies. Those policies, in combination with her own individual preferences to keep her personal and professional lives separate, make her rarely use her personal mobile phone at work. Liz is very computer savvy since she grabs a computer on wheels to look up reference information and enters patient data. Furthermore, she is not a laggard with mobile devices. She explains, "I like texting. But it's private versus work." Liz is not opposed to using a mobile device at work as long as it is not her own personal device. She suggests that her employer give her a phone that clearly identifies itself as a work phone. "We lock up our meds. We lock up stuff. Why can't we lock up our phones before we go? Keep it here. We don't have to carry it with us."

Liz suggests a way to be a personal/professional boundary segregator, yet still use a mobile device at work. In contrast to Anika, Liz hit roadblocks to using her mobile device at work from all three organizational levels. She did not want to be a victim of group-level policies, and she had a personal preference to be a segregator. Even if this organization made her aware of the acceptable use policy, the units abolished their differential rules, and the organization purchased mobiles for work-only use, Liz might still keep her personal and professional lives separate by using two different mobiles. Figure 2 depicts the interplay between all three levels of boundaries that Liz experienced in her mobile use. Each circle has a fixed, solid boundary and the individual's boundary engulfs the unit and organizational boundaries.

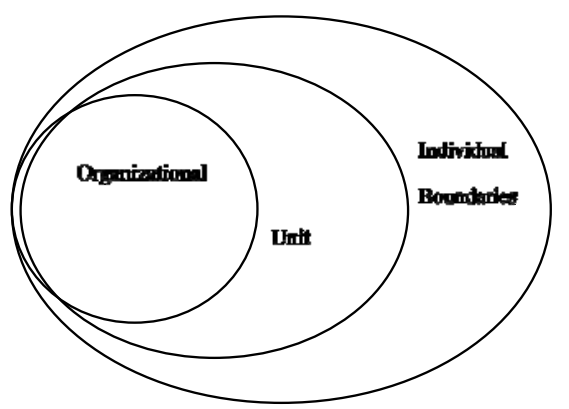

Figure 2. A segregator's personal mobile use at work

This figure illustrates that individual boundaries dominate over unit and organizational boundaries when workers have a strong desire to segregate work and personal life. Even if organizations make individuals aware of their BYOD policy and units abolish their policies, individual boundaries are still 
present. Note this is personal mobile device use at work and does not include people who have separate work mobiles.

\subsection{Limitations \& Future Research}

While this research raises some important boundary considerations that could be applicable to a wide variety of organizations, we should be cautious in generalizing beyond a hospital organization. Healthcare workers have specific communication requirements related to patient privacy that could affect how this population uses mobile devices. We did collect demographic data, and on the surface it appeared reasonably diverse, but there were pockets of nurses where our research did not reach. We also deliberately limited our analysis to focus on boundary issues involved in mobile device implementation.

This study paves the way for additional research related to information systems. One obvious extension is to use our findings to inform other BYOD implementation projects and systematically collect data. Studies can examine if implementation can be improved if organizations structure pilots broadly, communicate the policy effectively, improve connectivity, and address mobile plan costs.

Another area for future research is to explore how to get segregators more comfortable with the idea of using a mobile device to communicate at work. Our Liz example suggests that segregators will likely want two separate phones, but there could be other alternatives. For example, if a personal mobile device had an interface that clearly showed when people were switching from personal to professional use, segregators might find that acceptable.

The promise of BYOD and the use of mobile devices to support the provision of healthcare, is profound, but our data show that there are issues to consider. Implementation practices must consider involving members from multiple professions or they risk establishing a perspective that some professions can use their mobile and others cannot. Group/team level policies that restrict mobile device use will likely deter use, especially in professions that are hierarchical. Finally, individual-level boundary perceptions can have a big impact on whether individual adopt mobile communication devices and apps for work. Organizations need to be aware that simply giving people permission to use a mobile device at work is not enough to have them adopt the practice at a level where communication can be reliably improved.

\section{References}

[1] R. Ling, "Taken for Grantedness: The Embedding of Mobile Communication into Society”, MIT Press, Cambridge, MA, 2012.
[2] C. Middleton, R, Scheepers, and V.K. Tuunainen, "When Mobile is the Norm: Researching Mobile Information Systems and Mobility as Post-adoption Phenomena”, European Journal of Information Systems, 23, 2014, pp. 503-512.

[3] M. Bittman, J.E. Brown, and J. Wajcman, J. “The Mobile Phone, Perpetual Contact, and Time Pressure”, Work, Employment \& Society, 23, 2009, pp. 673-691.

[4] S. Sarker, X. Xiao, S. Sarker, and M. Ahuja, M. “Managing Employees' Use of Mobile Technologies to Minimize Work-life Balance Impacts”, MIS Quarterly Executive, 11, 2012, pp. 143-157.

[5] J. Wajcman, M. Bittman, and J.E. Brown, "Families Without Borders: Mobile Phones, Connectedness and Workhome Divisions”, Sociology, 42, 2008, pp. 635-652.

[6] J. Harris, B. Ives, and I. Junglas, "IT Consumerization: When Gadgets Turn Into Enterprise IT Tools”, MIS Quarterly Executive, 11, 2012, pp. 99-112.

[7] C. Sørensen, Enterprise Mobility: Tiny Technology with Global Impact on Work. Palgrave Mcmillan, NY, NY, 2011.

[8] K.K. Stephens and J.L. Ford, "Unintended Consequences of a Strategically Ambiguous Organizational Policy

Selectively Restricting Mobile Device Use at Work”, Mobile Media \&Communication, 4, 2016, pp. 186-201.

[9] K.K. Stephens and J.L. Ford, "Banning Mobile Devices: Workplace Policies that Selectively Exclude can Shape Crisis Communication”, In Proceedings of the 11th International ISCRAM Conference (pp. 277-281), University Park, PA, 2014.

[10] B.E. Ashforth, G.E., Kreiner, and M. Fugate, “All in a Day's Work: Boundaries and Micro Role Transitions”, The Academy of Management Review, 25, 2000, pp. 472-491.

[11] C.E. Nippert-Eng, Home and Work: Negotiating Boundaries Through Everyday Life, University of Chicago Press, Chicago, IL, 1996.

[12] J.B. Olson-Buchanan and W.R. Boswell, "Blurring Boundaries: Correlates of Integration and Segmentation Between Work and Nonwork”, Journal of Vocational Behavior, 68, pp. 432-445.

[13] J.N. Reyt and B.M. Wiesenfeld, "Seeing the Forest for the Trees: Exploratory Learning, Mobile Technology, and Knowledge Workers’ Role Integration Behaviors”, Academy of Management Journal, 58, 2015, pp. 739-762.

[14] N.P. Rothbard, K.W. Phillips, and T.L. Dumas, "Managing Multiple Roles: Work-Family Policies and Individuals' Desires for Segmentation”, Organization Science, 16, 2005, pp. 243-258.

[15] R. Ling and B. Yttri, "Hyper-coordination via Mobile Phones in Norway”, in J.E. Katz and M. Aakhus (Eds.), Perpetual Contact: Mobile Communication, Private Talk, 
Public Performance (pp. 139-169), Cambridge University Press, Cambridge, UK, 2002.

[16] V. Lo, R.C. Wu, D. Morra, L. Lee, and S. Reeves, "The Use of Smartphones in General and Internal Medicine Units: A Boon or Bane to the Promotion of Interprofessional Collaboration?”, Journal of Interprofessional Care, 26, 2012, pp. 276-282.

[17] B.M. Dala-Ali, M.A. Lioyd, and Y. AL-Abed, "The Uses of the iPhone for Surgeons”, Surgeon, 9, 2011, pp. 4448.

[18] Spok, Inc, "BYOD Trends in Healthcare: An Industry Snapshot”, 2015. Available : http://cloud.spok.com/IBAMER-BYOD-2015-Survey.pdf.

[19] PC Today, “Personal Devices at Work”, PC Today, 10, 2010, pp. 44-46.

[20] K.K. Stephens, A. Barrett, and M.L. Mahometa, "Organizational Communication in Emergencies: Using Multiple Channels and Sources to Combat Noise and Capture Attention”, Human Communication Research, 39, 2013, pp. 230-251.

[21] M. Mazmanian, W.J. Orlikowski, and J. Yates, “The Autonomy Paradox: The Implications of Mobile Email Devices for Knowledge Professionals”, Organization Science, 24, 2013, pp. 1337-1357.

[22] J.E. Bardram and C. Bossen, "Moving to Get Ahead: Local Mobility and Collaborative Work", in European Conference on Computer-Supported Work, Helsinki, Finland, 2003, pp. 355-374.

[23] S. Pink, J. Morgan, and A. Dainty, "Safety in Movement: Mobile Workers, Mobile Media”, Mobile Media \& Communication, 2, 2014, pp. 335-351.

[24] M.C. Reddy, D.W. McDonald, W. Pratt, and M.M. Shabot, "Technology, Work, and Information Flows: Lessons from the Implementation of a Wireless Alert Pager System”, Journal of Biomedical Informatics, 38(3), 2005, pp. 229-238.

[25] C. Nguyen, L.M. McElroy, M.M. Abecassis, J.L. Holl, \& D. Ladner, "The Use of Technology for Urgent Clinician to Clinician Communications: A Systematic Review of the Literature”, International Journal of Medical Informatics, 84, 2015, pp. 101-110.

[26] S.D. Quan, R.C. Wu, P.G., Rossos, et al., “It’s not About Pager Replacement: An In-depth Look at the Interprofessional Nature of Communication in Healthcare”, Journal of Hospital Medicine, 8, 2013, pp. 137-143.

[27] R. Wu, P. Rossos, S. Quan, S. Reeves, V. Lo, B. Wond, M. Cheung, and D. Morra, "An Evaluation of the Use of Smartphones to Communicate Between Clinicians: A Mixed-
Methods Study”, Journal of Medical Internet Research, 13, pp. e59-e68.

[28] L. Melby and R. Hellesø, "Introducing Electronic Messaging in Norwegian Healthcare: Unintended Consequences for Professional Collaboration”, International Journal of Medical Informatics, 83, 2014, pp. 343-353.

[29] T. Solvoll, J. Scholl, and G. Hartvigsen, "Physicians Interrupted by Mobile Devices in Hospitals: Understanding the Interaction Between Devices, Roles, and Duties”, Journal of Medical Internet Research, 15, 2013, pp. e56-e71.

[30] D.D. Luxton, R.A. McCann, N.E. Bush, M.C. Mishkind, and G.M. Reger, "mHealth for Mental Health: Integrating Smartphone Technology in Behavioral Healthcare”, Professional Psychology: Research and Practice, 42, 2011, pp. 505-512.

[31] E. Etchells, N.K. Adhikari, R. Wu, et al., "Real-time Automated Paging and Decision Support for Critical Laboratory Abnormalities”, BMJ Quality \& Safety, 20, 2011, pp. 924-930.

[32] E. Ozdalga, A. Ozdalga, and N. Ahuja, "The Smartphone in Medicine: A Review of Current and Potential use Among Physicians and Students", Journal of Medical Internet Research, 14, 2012, pp. 128-141.

[33] K. Charmaz, Constructing Grounded Theory: A Practical Guide Through Qualitative Analysis. Sage Publications, Thousand Oaks, CA, 2006.

[34] B. Glaser and A. Strauss, The Discovery of Grounded Theory, Transaction Publishers, Chicago, IL, 1967.

[35] ConsumerReports, (2015). Get the Best Cell Phone Plan for Your Family - And Save Up to \$1000 a year. Available: http://www.consumerreports.org/cro/news/2014/01/bestphone-plans-for-your-family-save-money/index.htm

[36] L. Kuokkanen and H. Leino-Kilpi, "Power and Empowerment in Nursing: Three Theoretical Approaches", Journal of Advanced Nursing, 31, 2000, pp. 235-241.

[37] M. Zwarenstein, K. Rice, L. Gotlib-Conn, C. Kenaszchuk, and S. Reeves, "Disengaged: A Qualitative Study of Communication and Collaboration Between Physicians and other Professions on General Internal Medicine Wards”, BMC Health Services Research, 2013, pp. 13-49.

[38] L. Bertalanffy, General System Theory. George Braziller, New York, NY, 1969.

[39] M.S. Poole, “Systems Theory”, in L.L. Putnam and D.K. Mumby (Eds.), The SAGE Handbook of Organizational Communication: Advances in Theory, Research, and Methods (3rd ed., pp. 49-74.), Sage, Thousand Oaks, CA, 2014 . 